\section{P103 HIV/STI TESTING AMONG MEN WHO HAVE SEX WITH MEN IN THE NETHERLANDS: ATTITUDES TOWARDS HOME-SAMPLING TESTING DURING THE COVID-19 PANDEMIC}

${ }^{1} \mathrm{H}$ Goense*, ${ }^{1} Y$ Evers, ${ }^{1,2} \mathrm{C}$ Hoebe, ${ }^{1,3} \mathrm{~N}$ Dukers-Muijrers. 'Department of Sexual Health Infectious Diseases and Environmental Health, South Limburg Public Health Service, Heerlen, The Netherlands; ' Department of Social Medicine and Medical Microbiology, School of Public Health and Primary Care (CAPHRI), Maastricht, The Netherlands; ${ }^{3}$ Department of Health Promotion, School of Public Health and Primary Care (CAPHRI), Maastricht University Medical Centre (MUMC+), Maastricht, The Netherlands

\subsection{6/sextrans-2021-sti.230}

Background The Covid-19 pandemic may have affected access to and uptake of HIV/STI testing among men who have sex with men (MSM) in the Netherlands and underpin the importance of remote sexual health care. Remote sexual health care, including home HIV/STI testing and remote consultation is increasingly appreciated as a necessary extension to regular care, to be able to reach out to MSM. While care providers recognize this need, evidence regarding attitudes among MSM towards using remote care is limited. Here, we present STI/HIV testing behaviors, attitudes and preferences, towards home-sampling during the Covid-19 pandemic, in MSM who (pre-pandemic) attended locationbased STI clinic care.

Methods Starting in November 2020, 417 MSM who attended one of several Dutch STI clinics before the Covid-19 pandemic were invited to participate in an online survey, Data collection is on-going. Descriptive statistics regarding testing for HIV/STI testing, of the first 148 participants (35.5\%) are presented. Attitude towards home-sampling testing is shown on a scale from negative (0) to positive (100).

Results Of participants (mean age 48) in the past 6 months, $66.4 \%$ (97/146) tested for STI and 61.6\% (90/146) tested for HIV. A home-sampling test for STI was used by $2.1 \%$ and $1.1 \%$ performed a home-sampling test for HIV. Participants reported that $1.5 \%$ of their male sex partners used home-sampling testing for HIV. Participants were positive towards home-sampling testing (mean=72.0, SD=33.6). When testing for HIV in the future $48.4 \%(60 / 124)$ would consider homesampling testing. Most of the participants (56.8\%) prefer remote consultations combined with a consultation at the STI clinic.

Conclusions Although home-sampling STI/HIV testing is rarely used by STI clinic visiting MSM, results show that MSM can be positive towards home-sampling testing for STI and HIV. Home-sampling testing and remote consultations might be solutions to extend sexual healthcare to continue reaching MSM.

\section{P104 APPLYING A NOVEL APPROACH TO SCOPING REVIEW INCORPORATING ARTIFICIAL INTELLIGENCE: MAPPING THE NATURAL HISTORY OF GONORRHOEA}

1J Whelan*, ${ }^{2} \mathrm{M}$ Ghoniem, ${ }^{2} \mathrm{~N}$ Médoc, ${ }^{3} \mathrm{M}$ Apicella, ${ }^{4} \mathrm{E}$ Beck. ${ }^{1}$ GSK Vaccines, Amsterdam, The Netherlands; ${ }^{2}$ Luxembourg Institute of Science and Technology, Belvaux, Luxembourg; ${ }^{3}$ University of lowa, lowa City, USA; ${ }^{4}$ GSK Vaccines, Wavre, Belgium

10.1136/sextrans-2021-sti.231
Background This scoping review presents the results of three distinct search strategies combined to identify and map the range of conditions (clinical presentations, complications, coinfections and health problems) associated with gonorrhoea infection. We also report use of a novel artificial-intelligence(AI)-assisted Medline search tool.

Methods To fully characterize the range of health outcomes associated with gonorrhoea, we combined a structured preliminary search with a traditional systematic search, then supplemented with the output of a novel AI-assisted Medline search to identify eligible literature.

Results We identified 189 health conditions associated with gonorrhoea infection from 107 unique references and 21 International Statistical Classification of Diseases and Related Health Problems Ninth and Tenth Revision (ICD 9/10) or Read codes. Pathogenic processes relating to these outcomes were also briefly summarized. The 189 identified health conditions were related to infection of the urogenital tract $(n=86)$, anorectal tract $(n=6)$ oropharyngeal tract $(n=5)$ and the eye $(n=14)$; and other conditions such as systemic $(n=61)$ and neonatal conditions $(n=7)$, psychosocial associations $(n=3)$, and co-infections $(n=7)$. The 107 unique references attained a Scottish Intercollegiate Guidelines Network (SIGN) score of $\geq 2++\quad(n=2), 2+(14 \quad[13 \%]), 2-(30 \quad[28 \%])$ and $3 \quad(45$ [42\%]), respectively. Remaining papers $(n=16)$ were reviews. Conclusions Through AI screening of Medline, we captured titles, abstracts, case reports and case series related to rare but serious health conditions. These outcomes might otherwise have been missed during a systematic search. The AI-assisted search provided a useful addition to traditional/manual literature searches especially when rapid results were required.

ACKNOWLEDGEMENT Business \&Decision Life Sciences (Coordinator: Julien Doornaert).

FUNDING GlaxoSmithKline Biologicals SA

\section{P106 INCREASED RISK OF SUBSEQUENT CHLAMYDIA INFECTION AMONG WOMEN NOT TESTED AT THE ANORECTAL ANATOMICAL LOCATION}

M Visser*, M Dvorakova, F van Aar, J Heijne. National Institute For Public Health and The Environment (RIVM), Bilthoven, The Netherlands

\subsection{6/sextrans-2021-sti.232}

Background Universal anorectal testing for Chlamydia trachomatis (chlamydia) among women is not recommended in many countries, while anorectal chlamydia infections are common. Missed anorectal infections might cause sequalae at the genital site if autoinoculation from the anorectum is possible, but evidence is limited. This study investigates the association between potentially missed anorectal infections and subsequent genital chlamydia infections in women, using not being tested at the anorectal site as a proxy for having a potentially missed anorectal infection

Methods We included all women with a repeat chlamydia test within one year (with at least a genital test) from the Dutch Sexual Health Centre (SHC) surveillance between 2014-2019. Multilevel logistic regression analyses were used to identify determinants of genital chlamydia infection at the repeat test, with anorectal testing at the previous chlamydia test as main determinant. 
Results A total of 40,217 women were included in the analyses, of whom $15.4 \%$ tested chlamydia positive genitally at their second test. Not being tested anorectally at the first test was an independent risk factor of genital chlamydia infection at the repeat test (aOR 1.24 [95\%CI 1.15-1.33]). This effect was in the same range as most other significant risk factors in the model: low education level, no condom use, STI symptoms and previous STI diagnosis. Young age $(<20$ years 2.67, [2.39-2.98]) and received partner notification (3.11, [2.913.31]) showed stronger effects. The findings were robust: correcting for interactions and a sensitivity analysis stratifying by chlamydia infection at first visit did not show significant differences in the aOR of not being tested anorectally at first test.

Conclusion The results are suggestive of an autoinoculation process from the anorectal to the genital anatomical site in women. To enhance chlamydia control, future studies on the role of extragenital testing and autoinoculation in chlamydia transmission are needed.

\section{P108 PREVALENCE AND EPIDEMIOLOGICAL FACTORS ASSOCIATED WITH TRICHOMONAS VAGINALIS INFECTION IN A US MULTICENTER STI CLINICAL STUDY}

J Kostera*. Abbott Laboratories, Des Plaines, USA

10.1136/sextrans-2021-sti.233

Trichomonas vaginalis (TV) is the most common, curable nonviral sexually transmitted infection (STI) in the world, yet the epidemiology of the infection in the United States is not well defined. The infection is often associated with preterm birth, low-birthweight infants, infertility, as well as a facilitator for increasing the risk for acquisition and transmission of human immunodeficiency virus (HIV). Risk factors for TV in women include older age, race, impoverished socioeconomic status, and multiple sexual partners. The epidemiology of TV in men is less delineated, as men are not routinely tested, and most infections are asymptomatic.

In this work, we present TV prevalence in conjunction with Mycoplasma genitalium, Chlamydia trachomatis, and Neisseria gonorrhoeae prevalence with socio-demographic epidemiological data associated with patients enrolled in a multicenter STI study. Prevalence data was generated using the automated multiplex qualitative CE marked assay, Alinity m STI. The study included symptomatic and asymptomatic patients including a cohort of individuals who have been previously diagnosed with an STI seeking treatment at public health clinics and primary care offices within the United States.

The prevalence of TV in women was determined to be $12.3 \%$ and $0.6 \%$ in men. When stratified by ethnicity and prior STI history in this cohort, the positivity rate of TV in African American women was $22.3 \%$ compared to $1.6 \%$ in White women. Furthermore, in this data set, African American women 40 and older had the highest TV positivity rate at $26.5 \%$ compared to $22.9 \%$ for those $30-39$, and $21.1 \%$ for those less than 29 years old. Those who reported not being diagnosed with a prior STI had a TV prevalence of $16.7 \%$ for African American women compared to $1.3 \%$ of White women. In this study, the overall prevalence of Chlamydia trachomatis was 9.8\%, Neisseria gonorrhoeae was $2.4 \%$, and Mycoplasma genitalium was $11.6 \%$.

\section{P112 REMOVING BARRIERS TO HIV SCREENING IN A COMMUNITY-BASED PEDIATRIC EMERGENCY DEPARTMENT}

${ }^{1} \mathrm{M}$ Gaines*, '1J Wiggins, 'B Sierzant, ${ }^{2,3}$ B Coleman, ${ }^{1,2} \mathrm{~W}$ Hannah. ${ }^{1}$ Memorial Health University Medical Center, Savannah, USA; ${ }^{2}$ Mercer University School of Medicine, Savannah, USA; ${ }^{3}$ Georgia Emergency Physicians Specialists, Savannah, USA

\subsection{6/sextrans-2021-sti.234}

Background Memorial University Medical Center conducts opt-out HIV screening in the Emergency Department (ED) for patients aged $\geq 13$. After gaining consent, an electronic algorithm automatically orders an HIV test with the diagnostic evaluation for patients who meet CDC guidelines for HIV screening. Unfortunately, no pediatric patient aged 13-18 received HIV screening despite our out-opt testing protocol being in place since 2016 .

Approach Through a systematic analysis, implicit bias to gain consent was repeatedly identified as the most common barrier to HIV screening in this population. Pediatric leadership developed strategies to improve HIV screening, including education of parents and staff, on-site support for staff and parents/ guardians, pediatric patient counseling, and adding HIV screening to syphilis tests. Despite these interventions, HIV screening in the pediatric population only improved about $37 \%$ from January 2017 through November 2020. As a final strategy HIV screening was added to the sexually transmitted infection (STI) order set for patients who met CDC guidelines for STI. Pediatric ED HIV education to clinical staff continued, specifically emphasizing the high prevalence of HIV in our community.

Outcomes Persistence to drive change increased HIV screening by $61 \%$ from July 2020 to December 2020. Strategies producing the greatest impact were the inclusion of HIV testing in the standard STI panel and focused pediatric ED nurse education/support. As a result, current HIV screening in our pediatric patients aged $13-18$ is now $88 \%$ of those who meet CDC guidelines to be screened.

Significance Up to $20 \%$ of HIV diagnoses occur during adolescence making screening in this age group imperative. Removing implicit bias from HIV screening is difficult. However, continued persistence and automated testing protocols can lead to increases in pediatric ED HIV screening. Because of our high HIV screening rate, many pediatric patients will have early identification of HIV and linkage to care.

\section{P113 ACCEPTABILITY AND PREFERENCES FOR USING A NOVEL DEVICE TO SELF-COLLECT BLOOD SPECIMENS FOR HIV PRE-EXPOSURE PROPHYLAXIS (PREP) LABORATORY MONITORING}

${ }^{1,2} \mathrm{C}$ Cannon*, ${ }^{1,2} \mathrm{C}$ Malinski, ${ }^{1,2} \mathrm{M}$ Golden. 'University of Washington, Seattle, USA; ${ }^{2}$ Public Health-Seattle and King County, Seattle, USA

10.1136/sextrans-2021-sti.235

Home-based PrEP monitoring (HBPM) has the potential to improve PrEP access and retention but is hampered by difficulties collecting blood, which typically involves painful fingersticks that yield insufficient specimen for quantitative syphilis serologies. We evaluated the use of the Tasso OnDemand ${ }^{\mathrm{TM}}$, a device that facilitates relatively painless self-collection of 